\title{
A FINITENESS CONDITION ON REGULAR LOCAL OVERRINGS OF A LOCAL DOMAIN
}

\author{
BERNARD JOHNSTON
}

\begin{abstract}
The local factorization theorem of Zariski and Abhyankar implies that between a given pair of 2-dimensional regular local rings, $S \supseteq R$, having the same quotient field, every chain of regular local rings must be finite. It is shown in this paper that this property extends to every such pair of regular local rings, regardless of dimension. An example is given to show that this does not hold if "regular" is replaced by "Cohen-Macaulay," by "normal," or by "rational singularity." More generally, it is shown that the set $R(R)$ of $n$ dimensional regular local rings birationally dominating a given $n$-dimensional local domain, $R$, and ordered by containment, satisfies the descending chain condition. An example is given to show that if $R$ is regular the two examples of minimal elements of $R(R)$ given by J. Sally do not exhaust the set of minimal elements of $R(R)$.
\end{abstract}

1. Introduction. The local factorization theorem of Zariski and Abhyankar states that if $R$ and $S$ are 2-dimensional regular local rings with $R \subseteq S \subseteq$ quotient field of $R$, then $S$ is obtainable from $R$ by a unique finite sequence of quadratic transforms (see below for definitions) (cf. [10, Lemma, p. 538; 1, Theorem 3; and 9, $\$ 2])$. Sally $[\mathbf{8}$, Corollary 4.5] and Shannon [9, Example 3.2] showed that a straightforward generalization of this result does not extend to higher dimensions, even if monoidal transforms are considered in place of quadratic transforms. Shannon was able to show that no infinite ascending chain of regular local rings could exist between two higher-dimensional regular local rings, of the same dimension [9, Proposition 5.1]. This paper shows that no infinite descending chain of such rings can occur and so, using Shannon's result, we are able to conclude, as Corollary 4.10 below, that a strong finiteness condition continues to hold between $R$ and $S$, even if their mutual dimension is greater than 2; namely, every chain of regular local rings between them must be finite. In fact, we obtain, as Theorem 4.6, the somewhat more general statement that the $n$-dimensional regular local rings birationally containing a fixed local domain of the same dimension must satisfy the descending chain condition (d.c.c). As an application of these ideas, we note in Example

Received by the editors August 13, 1985 and, in revised form, February 25, 1986. The results in this paper were presented to the eight-hundred and twenty-third meeting of the American Mathematical Society at the University of Missouri, Columbia, on November 1, 1985.

1980 Mathematics Subject Classification(1985 Revision). Primary 13H05; Secondary 13E99, $14 \mathrm{E} 40$.

Key words and phrases. Regular local rings, birational, descending chain condition, domination, monoidal transform.

This paper forms part of the author's Ph.D. thesis written at Purdue University under the supervision of Professor Williarn Heinzer. The author wishes to thank Professor Heinzer and Professor Craig Huneke for many stimulating conversations and suggestions. Thanks are also due Professor David Lantz for a thorough reading of the manuscript and many helpful comments. 
5.4 that the two types of minimal regular local overrings of a given $n$-dimensional regular local ring $(n>2)$ given by J. Sally cannot be the only such examples.

In Examples 4.9 and 5.2 we note that such strong finiteness conditions do not hold among some local domains which are more general than regular local rings.

The technical heart of the paper is contained in $\S 3$, in which the crucial result is Theorem 3.2. Preliminaries are given in $\S 2$, while $\S 4$ contains the main results, and $\S 5$ contains some extensions of these.

2. Preliminaries. If $R$ is a quasilocal ring (i.e., $R$ has a unique maximal ideal), we use the symbol $(R, \mathcal{M})$ to indicate explicitly that $\mathcal{M}$ is the unique maximal ideal of $R$. We use the word "local" to indicate a Noetherian quasilocal ring throughout. We use the symbol $\subset$ to indicate strict inclusion. By a regular local ring (RLR) we mean a local domain whose unique maximal ideal can be generated by $n$ elements, where $n$ is the Krull dimension of $R$, which we denote by $\operatorname{dim} R$. The height of a prime $P$ is denoted ht $P$. If $(R, \mathcal{M})$ is a RLR, we use the phrase "regular system of parameters" for $\mathcal{M}$ to mean a minimal generating set for $\mathcal{M}$.

We denote the quotient field of any domain $R$ by q.f. $(R)$. We say that $S$ and $R$ are "birational" if q.f. $(S)=$ q.f. $(R)$. We say that " $(S, \mathcal{N})$ dominates $(R, \mathcal{M})$ " if $S \supseteq R$ and $\mathcal{N} \cap R=\mathcal{M}$. We use the symbol $S>R$ to indicate that $S$ dominates $R$ and $S$ is birational to $R$. If $(S, \mathcal{N})>(R, \mathcal{M})$ are quasilocal domains, then the field $S / \mathcal{N}$ contains the field $R / \mathcal{M}$ canonically. We denote the transcendence degree of $S / \mathcal{N}$ over $R / \mathcal{M}$ by restrdeg $R$, for "residual transcendence degree of $S$ over $R$."

Note 2.1. Let $(S, \mathcal{N})$ and $(R, \mathcal{M})$ be two local domains of the same dimension. Then $S>R$ if and only if $R \subseteq S \subseteq$ q.f. $(R)$.

PROOF. $(\Rightarrow)$ This is by definition of $S>R$.

$(\Leftarrow)$ We need only see that $\mathcal{N} \cap R=\mathcal{M}$. Certainly $\mathcal{N} \cap R \subseteq \mathcal{M}$. If $\mathcal{N} \cap R \subset \mathcal{M}$ then $\operatorname{dim} R_{\mathcal{N} \cap R}<\operatorname{dim} R=\operatorname{dim} S$, and $R_{\mathcal{N} \cap R} \subset S \subset$ q.f. $(R)=$ q.f. $\left(R_{\mathcal{N} \cap R}\right)$. This contradicts the fact that any local domain between an $n$-dimensional local domain and its quotient field must itself have dimension $\leq n$ (see [9, Lemma 5.3]).

If $(R, \mathcal{M})$ is a RLR, several specific constructions of RLR's $S$ such that $S>R$ are of particular interest. Namely, a "monoidal transform" of $R$ is a ring of the form $S:=R\left[x_{2} / x_{1}, x_{3} / x_{1}, \ldots, x_{i} / x_{1}\right]_{Q}$, where $\left\{x_{1}, \ldots, x_{i}\right\}$ is part of a regular system of parameters for $\mathcal{M}$, and $Q$ is a maximal ideal of $R\left[x_{2} / x_{1}, \ldots, x_{i} / x_{1}\right]$ whose height equals $\operatorname{dim} R$. A "simple monoidal transform" of $R$ is a monoidal transform in which only one element is adjoined to $R$, e.g., $S=R\left[x_{2} / x_{1}\right]_{Q}$. A "quadratic transform" of $R$ is a monoidal transform of $R$ in which as many elements as possible have been adjoined to $R$, i.e., $S=R\left[x_{2} / x_{1}, \ldots, x_{n} / x_{1}\right]_{Q}$, where $\left\{x_{1}, \ldots, x_{n}\right\}$ constitutes all of a regular system of parameters for $\mathcal{M}$, i.e., $n=\operatorname{dim} R$.

If $(R, \mathcal{M})$ is a local ring and $x \in R \backslash\{0\}$, then $x \notin \bigcap_{i=0}^{\infty} \mathcal{M}^{i}$ by Krull's Intersection Theorem [11, Corollary 2, p. 217]. Therefore there exists $r \geq 0$ such that $x \in$ $\mathcal{M}^{r} \backslash \mathcal{M}^{r+1}$. We define a function on $R$, "ord," by $\operatorname{ord}(x):=r$ (ord $\left.0:=\infty\right)$. We extend this to q.f. $(R)$ by $\operatorname{ord}(a / b):=\operatorname{ord}(a)-\operatorname{ord}(b)$. If in addition $R$ is regular then this order function is actually a valuation, i.e., we have ord $(a b)=\operatorname{ord}(a)+\operatorname{ord}(b)$ and $\operatorname{ord}(a+b) \geq \min \{\operatorname{ord}(a), \operatorname{ord}(b)\}[12$, Corollary 1, p. 302]. Among valuation rings, we reserve the symbol DVR for the valuation domains which are rank 1 and discrete, where "rank" means Krull dimension. We note that the DVR's contained in a given field are maximal elements among the set of quasilocal domains contained in that field and ordered by domination [3, Kriterium 2, p. 102]. 
By a "chain of RLR's between $R$ and $S$ " we mean a nonempty set $\left\{S_{\lambda}\right\}$ of distinct birational RLR's linearly ordered by domination and such that $R<S_{\lambda}<S$ for all $\lambda$. Given an infinite descending sequence, $\left(S_{0}, \mathcal{M}_{0}\right) \supseteq\left(S_{1}, \mathcal{M}_{1}\right) \supseteq \cdots$ of quasilocal rings such that $S_{i}$ dominates $S_{i+1}$ for all $i$, we denote the intersection by " $\left(S_{\infty}, \mathcal{M}_{\infty}\right)$ ". With regard to this $S_{\infty}$ we have the following lemma.

LEMMA 2.2. $\left(S_{\infty}, \mathcal{M}_{\infty}\right)$ is a quasilocal ring.

Proof. If $\alpha \in \mathcal{M}_{\infty} \subseteq \mathcal{M}_{0}$ then $\alpha$ is not a unit in $S_{\infty}$, for if it were, we would have $\alpha^{-1} \in S_{\infty} \subseteq S_{0}$ so $\alpha$ would be a unit in $S_{0}$, a contradiction. On the other hand, if $\alpha \in S_{\infty} \backslash \mathcal{M}_{\infty}$ then $\alpha \in S_{i} \backslash \mathcal{M}_{i}$ for some $i$ and hence in $S_{j} \backslash \mathcal{M}_{j}$ for all $j$; so $\alpha^{-1}$ is in every $S_{j}$ and hence in $S_{\infty}$. Thus the set of nonunits in $S_{\infty}$ forms the ideal $\mathcal{M}_{\infty}$.

3. Infinite descending chains of RLR's. This section contains the main technical result of this paper, Theorem 3.2, which states that the quotient field of the intersection of a certain chain of regular local rings, all having the same quotient field, $K$, is strictly smaller than $K$. The following example is provided as motivation.

EXAMPLE 3.1. Let

$$
\left(S_{0}, \mathcal{M}_{0}\right):=\left(k[X, Y]_{(X, Y)},(X, Y) k[X, Y]_{(X, Y)}\right),
$$

where $k$ is any field, and $X$ and $Y$ are indeterminates. Let

$$
\left(S_{i}, \mathcal{M}_{i}\right):=\left(k\left[X, X^{i} Y\right]_{\left(X, X^{i} Y\right)},\left(X, X^{i} Y\right) k\left[X, X^{i} Y\right]_{\left(X, X^{i} Y\right)}\right) .
$$

Clearly $\left(S_{0}, \mathcal{M}_{0}\right) \supset\left(S_{1}, \mathcal{M}_{1}\right) \supset \cdots$, and since the matrix $\left(\begin{array}{ll}1 & 0 \\ i & 1\end{array}\right)$ has determinant 1 , this is a strictly descending chain of RLR's with the same quotient field, $K=$ $k(X, Y)$. In this example there is no residue field extension, i.e., $S_{i} / \mathcal{M}_{i}=k$ for all $i$. We have $S_{\infty}=k[X]_{(X)}$, so q.f. $\left(S_{\infty}\right)=k(X)$ is strictly smaller than q.f. $\left(S_{i}\right)=$ $k(X, Y)$.

That the behavior exhibited in this simple example extends to the general case is the gist of the following theorem, and its proof.

THEOREM 3.2. Suppose that $\left(S_{0}, \mathcal{M}_{0}\right)>\left(S_{1}, \mathcal{M}_{1}\right)>\cdots$ is an infinite strictly descending sequence of regular local rings, all having the same quotient field, $K$, and the same dimension, $n$. Suppose, in addition, that the residue fields eventually stabilize, i.e., there exists $N \geq 0$ so that $S_{i} / \mathcal{M}_{i}=S_{i+1} / \mathcal{M}_{i+1}$ for all $i \geq N$. Then the quotient field of the intersection, $\left(S_{\infty}, \mathcal{M}_{\infty}\right)$, is strictly contained in $K$.

Before proceeding with the proof we note a few remarks and lemmas.

REMARKS 3.3. (1) Deleting all but a cofinal set of rings will not change the intersection so, when convenient, we may ignore all but a cofinal set of rings in the chain. In particular, we may assume $S_{0} / \mathcal{M}_{0}=S_{1} / \mathcal{M}_{1}=\cdots=k$.

(2) The theorem is vacuously true if $n=1$ since a 1 -dimensional RLR is a DVR (see [5, Theorem 37] and [6, Theorem 12.4]) and any chain of DVR's of the form $S_{0}>S_{1}>\cdots$ can occur only if $S_{0}=S_{1}=\cdots$, since DVR's are maximal among the set of quasilocal domains within their quotient field (see §2). So we assume without loss of generality that $n \geq 2$ throughout this section. 
LEMMA 3.4. There is a cofinal subset $C$ of the positive integers for which the following is true: for $i$ in $C$ the $k$-vector spaces $V_{i, j}:=\left(\mathcal{M}_{j}+\mathcal{M}_{i}^{2}\right) / \mathcal{M}_{i}^{2}$ are equal for all $j$ in $C$ with $j>i$, and $\operatorname{dim}_{k} V_{i, j}=\operatorname{dim}_{k} V_{i^{\prime}, j}=: d$ whenever $i, i^{\prime}, j$ are in $C$ with $j>i$ and $j>i^{\prime}$. Moreover, $d<n$ and the theorem is true if $d=0$.

ProOf. We will construct the set $C$ by successively removing some of the rings in the original chain by the process described below and then renumbering. The rings in the chain which results, a subchain of the original chain, will be the rings indexed by $C$.

For $i=0$, then for $i=1$, etc., consider the descending sequence of $k$-vector spaces:

$$
V_{i, i}:=\mathcal{M}_{i} / \mathcal{M}_{i}^{2} \supseteq V_{i, i+1}:=\left(\mathcal{M}_{i+1}+\mathcal{M}_{i}^{2}\right) / \mathcal{M}_{i}^{2} \supseteq V_{i, i+2}:=\left(\mathcal{M}_{i+2}+\mathcal{M}_{i}^{2}\right) / \mathcal{M}_{i}^{2} \supseteq \cdots
$$

Since

$$
n=\operatorname{dim}_{k} V_{i, i} \geq \operatorname{dim}_{k} V_{i, i+1} \geq \operatorname{dim}_{k} V_{i, i+2} \geq \cdots \geq 0,
$$

there exists $d_{i}, n \geq d_{i} \geq 0$, and there exists $r_{i}>i$ so that

$$
\begin{aligned}
d_{i} & =\operatorname{dim}_{k} V_{i, r_{i}}=\operatorname{dim}_{k}\left(\mathcal{M}_{r_{i}}+\mathcal{M}_{i}^{2}\right) / \mathcal{M}_{i}^{2} \\
& =\operatorname{dim}_{k}\left(\mathcal{M}_{r_{i}+1}+\mathcal{M}_{i}^{2}\right) / \mathcal{M}_{i}^{2}=\operatorname{dim}_{k} V_{i, r_{i}+1}=\cdots .
\end{aligned}
$$

Then removing $S_{j}, i<j<r_{i}$, and renumbering $r_{i}+l:=i+l+1, l \geq 0$, we have $d_{i}=\operatorname{dim}_{k} V_{i, j}$ for all $j>i$.

Notice that if $j>i \geq 1$ there exists a surjective $k$-vector space map

$$
\begin{aligned}
\Theta: V_{i, j} & =\left(\mathcal{M}_{j}+\mathcal{M}_{i}^{2}\right) / \mathcal{M}_{i}^{2} \cong \mathcal{M}_{j} / \mathcal{M}_{j} \cap \mathcal{M}_{i}^{2} \\
& \rightarrow \mathcal{M}_{j} / \mathcal{M}_{j} \cap \mathcal{M}_{i-1}^{2} \cong\left(\mathcal{M}_{i}+\mathcal{M}_{i-1}^{2}\right) / \mathcal{M}_{i=1}^{2}=V_{i-1, j},
\end{aligned}
$$

so $d_{i}=\operatorname{dim}_{k} V_{i, j} \geq \operatorname{dim}_{k} V_{i-1, j}=d_{i-1}$. Thus we have $0 \leq d_{0} \leq d_{1} \leq \cdots \leq n$, since $d_{i} \leq \operatorname{dim}_{k} V_{i, i}=n$ for all $i$, so there exists $i^{\prime} \geq 0$ such that $d_{i^{\prime}}=d_{i^{\prime}+1}=\cdots=: d$. If $i>0$ we delete $S_{j}, 0 \leq j \leq i^{\prime}-1$, and renumber $i^{\prime}+l:=l, l \geq 0$, to conclude (in any case) that $\operatorname{dim} V_{i, j}=d$ whenever $j>i \geq 0$ and moreover, $n \geq d \geq 0$.

Suppose $d=n$. Then in particular, $\operatorname{dim}_{k}\left(\mathcal{M}_{1}+\mathcal{M}_{0}^{2}\right) / \mathcal{M}_{0}^{2}=n$, so there exist $\eta_{1}, \ldots, \eta_{n} \in \mathcal{M}_{1}$ such that $\bar{\eta}_{1}, \ldots, \bar{\eta}_{n}$ (where "-" indicates image $\bmod \mathcal{M}_{0}^{2}$ ) are linearly independent over $k$. It follows that $\bar{\eta}_{1}, \ldots, \bar{\eta}_{n}$ generate $\mathcal{M}_{0}\left(\bmod \mathcal{M}_{0}^{2}\right)$ over $k$, and hence (by Nakayama's Lemma $[\mathbf{5}, 1 . \mathrm{N}]$ ) that $\eta_{1}, \ldots, \eta_{n}$ generate $\mathcal{M}_{0}$. But then $\mathcal{M}_{1} S_{0}=\mathcal{M}_{0}$, and so (by Zariski's Main Theorem [2, Proposition 14]) $S_{0}=S_{1}$, which contradicts our assumption that the chain is strictly decreasing. So $d \leq n-1$.

On the other hand, suppose $d=0$. Then $\operatorname{dim}_{k}\left(\mathcal{M}_{i}+\mathcal{M}_{i-1}^{2}\right) / \mathcal{M}_{i-1}^{2}=0$, giving $\mathcal{M}_{i} \subseteq \mathcal{M}_{i-1}^{2} \subseteq \mathcal{M}_{i-2}^{4} \subseteq \cdots \subseteq \mathcal{M}_{0}^{2 i}$ for $i \geq 1$, so $\mathcal{M}_{\infty}=\bigcap_{i=0}^{\infty} \mathcal{M}_{i} \subseteq \bigcap_{i=1}^{\infty} \mathcal{M}_{0}^{2 i}=0$ (by Krull's Intersection Theorem [11, Corollary 2, p. 217]). Hence q.f. $\left(S_{\infty}\right)$ is strictly smaller than q.f. $\left(S_{0}\right)$, and the theorem is true.

REMARK 3.5. The point of this lemma is this. In proving Theorem 3.2 we need to consider, by Remark 3.3(1), only a cofinal subset of the original chain of rings. Letting $C$ be this cofinal subset, this lemma allows us to assume, in the proof of Theorem 3.2, that the $k$-vector spaces $V_{i, j}$ are equal whenever $j>i, i$ fixed, that they have the same dimension, $d$, for all $i$, and that $1 \leq d \leq n-1$. We make these assumptions throughout the remainder of this section. 
LEMMA 3.6. If $x_{i 1}, \ldots, x_{i d} \in \mathcal{M}_{i+1}$ are elements whose images in $V_{i, i+1}$ are a basis of $V_{i, i+1}$, then in fact their images in $V_{j, j+1}$ are a basis of $V_{j, j+1}$ for all $j$, $0 \leq j \leq i$. In particular, it follows that $\left\{x_{i 1}, \ldots, x_{i d}\right\}$ is part of a regular system of parameters for $\mathcal{M}_{j}, 0 \leq j \leq i$.

Proof. By Remark 3.5 all the $V_{i, j}$ have the same dimension, $d$, so the surjection $\theta: V_{i, i+1} \rightarrow V_{i-1, i+1}$ given in the proof of Lemma 3.4 is actually a $k$-isomorphism which takes the images of $x_{i 1}, \ldots, x_{i d}$ in $V_{i, i+1}$ to their images in $V_{i-1, i+1}$. Thus these images are a basis for $V_{i-1, i+1}$, which equals $V_{i-1, i}$. This proves the statement for $j=i-1$ and it is clear we may iterate to achieve the result for all $j$. The last statement follows because, for all $i, V_{i, i+1} \subset V_{i, i}$.

The following proposition is the key reduction needed for computational purposes.

Proposition 3.7. Let $d$ be as in Remark 3.5. For all $i \geq 0$ there exists $\left\{x_{i 1}, \ldots, x_{i d}\right\}$, part of a regular system of parameters for $\mathcal{M}_{i}$, such that for each $j, 0 \leq j \leq i$, there exists $y_{j 1}, \ldots, y_{j, n-d} \in \mathcal{M}_{j}$, with $\left\{x_{i 1}, \ldots, x_{i d}, y_{j 1}, \ldots, y_{j, n-d}\right\}$ a regular system of parameters for $\mathcal{M}_{j}$, and such that $y_{j l} \in \mathcal{M}_{0}^{j+1}, 1 \leq l \leq n-d$.

In particular, this gives, for each $i \geq 0$, a regular system of parameters $\left\{x_{i 1}, \ldots\right.$, $\left.x_{i d}, y_{i 1}, \ldots, y_{i, n-d}\right\}$ for $\mathcal{M}_{i}$ such that $\bar{x}_{i 1}, \ldots, \bar{x}_{i d}$ are linearly independent $\left(\bmod \mathcal{M}_{0}^{2}\right)$ over $k$, and $y_{i 1}, \ldots, y_{i, n-d} \in \mathcal{M}_{0}^{i+1}$.

PROOF. Fix $i$ and choose fixed $x_{i 1}, \ldots, x_{i d} \in \mathcal{M}_{i+1}$ whose images in $V_{i, i+1}$ are a basis. By Lemma 3.6, $\left\{x_{i 1}, \ldots, x_{i d}\right\}$ is part of a regular system of parameters for $\mathcal{M}_{j}, 0 \leq j \leq i$. We will show by induction on $j$ that for all such $j$ there exists $\left\{y_{j 1}, \ldots, y_{j, n-d}\right\}$ as in the statement.

Case $j=0$. Clear.

Case $j=1$. Let $z_{11}, \ldots, z_{1, n-d}$ be any elements of $\mathcal{M}_{0}$, so that $\left\{x_{i 1}, \ldots, x_{i d}\right.$, $\left.z_{11}, \ldots, z_{1, n-d}\right\}$ forms a regular system of parameters for $\mathcal{M}_{1}$. Then for each $l, 1 \leq$ $l \leq n-d$, we may write, in $V_{0,1}, \bar{z}_{1, l}=\bar{\alpha}_{1 l} \bar{x}_{i 1}+\cdots+\bar{\alpha}_{d l} \bar{x}_{i d}$, where $\bar{\alpha}_{s l} \in k$, $1 \leq s \leq d$. Picking preimages of $\bar{\alpha}_{s l}$ in $S_{1}$ we have $z_{1 l}=\alpha_{1 l} x_{i 1}+\cdots+\alpha_{d l} x_{i d}+y_{1 l}$, where $y_{1 l} \in \mathcal{M}_{0}^{2} \cap S_{1}$. Then

$$
\left(x_{i 1}, \ldots, x_{i d}, y_{11}, \ldots, y_{1, n-d}\right) S_{1}=\left(x_{i 1}, \ldots, x_{i d}, z_{i 1}, \ldots, z_{i, n-d}\right) S_{1}=\mathcal{M}_{1},
$$

so $\left\{x_{i 1}, \ldots, x_{i d}, y_{11}, \ldots, y_{1, n-d}\right\}$ gives the desired regular system of parameters for $\mathcal{M}_{n}$.

Case $1<j \leq i$. We apply the induction hypothesis to assume that $\left\{x_{i 1}, \ldots, x_{i d}\right.$, $\left.y_{j-1,1}, \ldots, y_{j-1, n-d}\right\}$ is a regular system of parameters for $\mathcal{M}_{j-1}$ so that $y_{j-1,1}, \ldots$, $y_{j-1, n-d} \in \mathcal{M}_{0}^{j}$.

We now apply the case $j=1$ to the truncated descending chain starting at $S_{j-1}$. That case implies that we can find $z_{j 1}, \ldots, z_{j, n-d} \in \mathcal{M}_{j}$ such that $x_{i 1}, \ldots, x_{i d}$, $z_{j 1}, \ldots, z_{j, n-d}$ form a regular system of parameters for $\mathcal{M}_{j}$ and such that each $z_{j l}$ is in $\mathcal{M}_{j-1}^{2}, 1 \leq l \leq n-d$. Thus each $z_{j l}$ can be written as a homogeneous polynomial of degree two in $x_{i 1}, \ldots, x_{i d}, y_{j-1,1}, \ldots, y_{j-1, n-d}$ with coefficients in $S_{j-1}$. Since we have equality of residue fields, any monomial in this polynomial whose coefficient is a unit in $S_{j-1}$ can be written as the sum of two monomials, one with coefficient a unit in $S_{j}$, and the other with a coefficient in $\mathcal{M}_{j-1}$. After performing this process for each monomial in $z_{j l}$, we collect all monomials whose 
coefficient is a unit in $S_{j}$, and call their sum $p_{1 l}$, while the sum of the monomials whose coefficient is in $\mathcal{M}_{j-1}$ we call $q_{1 l}$. Of course, $z_{j l}=p_{1 l}+q_{1 l}$ and $q_{1 l} \in \mathcal{M}_{j-1}^{3}$.

Hence $q_{1 l}$ can be written as a homogeneous polynomial of degree three in $x_{i 1}, \ldots, x_{i d}, y_{j-1,1}, \ldots, y_{j-1, n-d}$ and so we may repeat the above process to write $q_{1 l}=p_{2 l}+q_{2 l}$, where $p_{2 l}$ is a sum of monomials (of degree three) whose coefficients are units in $S_{j 1}$ and $q_{2 l} \in \mathcal{M}_{j-1}^{4}$. Now $z_{j l}=p_{1 l}+p_{2 l}+q_{2 l}$. We iterate until reaching $z_{k l}=p_{1 l}+p_{2 l}+\cdots+p_{j-1, l}+q_{j-1, l}$. Note that $q_{j-1, l} \in \mathcal{M}_{j-1}^{j+1} \subseteq \mathcal{M}_{0}^{j+1}$.

Let $p_{l}:=p_{1 l}+\cdots+p_{j-1, l}$. Then $p_{l}$ is a polynomial in $x_{i 1}, \ldots, x_{i d}, y_{j-1,1}, \ldots$, $y_{j-1, n-d}$ in which every monomial has degree at least two and every coefficient is a unit in $S_{j}$. But now $p_{l}$ can be rewritten as $p_{l}=p_{l}^{\prime}+p_{l}^{\prime \prime}$ where $p_{l}^{\prime}$ is the sum of those terms of $p_{l}$ which involve only the $x_{i}$ 's, and $p_{l}^{\prime \prime}$ is the sum of those terms which involve at least one $y_{j-1, r}$. We now have $z_{j l}=p_{l}^{\prime}+p_{l}^{\prime \prime}+q_{j-1, l}$. Set $y_{j l}:=z_{j l}-p_{l}^{\prime}$. Since every term in $p_{l}^{\prime}$ involves at least two $x_{i r}$ 's and has a coefficient which is a unit in $S_{j}$, we see that $p_{l}^{\prime} \in \mathcal{M}_{j}^{2}$. Therefore, since $\left\{x_{i 1}, \ldots, x_{i d}, z_{j 1}, \ldots, z_{j, n-d}\right\}$ is a regular system of parameters for $\mathcal{M}_{j}$, so is $\left\{x_{i 1}, \ldots, x_{i d}, y_{j 1}, \ldots, y_{j, n-d}\right\}$.

It remains to see that each $y_{j l} \in \mathcal{M}_{0}^{j+1}$. But we have $y_{j l}=z_{j l}-p_{l}^{\prime}=p_{l}^{\prime \prime}+q_{j-1, l}$, $q_{j-1, l} \in \mathcal{M}_{0}^{j+1}$, so we need only see that $p_{l}^{\prime \prime} \in \mathcal{M}_{0}^{j+1}$. This is clear since each $y_{j-1, r} \in \mathcal{M}_{0}^{j}$ and each $x_{i r} \in \mathcal{M}_{0}$, so each term in $p_{l}^{\prime \prime}$ is in $\mathcal{M}_{0}^{j+1}$.

The technique employed in the latter part of the proof of Proposition 3.7 was essentially the expansion of elements in $\mathcal{M}_{i}$ as power series with respect to a fixed regular system of parameters for $\mathcal{M}_{i}$. Had $S_{i}$ contained a coefficient field, for all $i$, then we would have been able to actually expand these elements as power series by passing to the completion and using Cohen's Structure Theorem [6, Theorem (31.1)]. The far clumsier technique of the text seems necessary in order to avoid the assumption of the existence of a coefficient field. A similar technique is also used in the proof of Lemma 3.8.

LEMMA 3.8. For each positive integer $i$, and for each $j, 1 \leq j \leq i$, let $\left\{x_{i 1}, \ldots, x_{i d}, y_{j 1}, \ldots, y_{j, n-d}\right\}$ be a regular system of parameters for $\mathcal{M}_{j}$ as in Proposition 3.7. If $z \in \mathcal{M}_{0}^{j} \cap \mathcal{M}_{j}$, then $z$ can be written $z=f+g$, where $f$ is a homogeneous polynomial of degree $j$ in $x_{i 1}, \ldots, x_{i d}$ whose nonzero coefficients are units in $S_{0}$, and where $g \in \mathcal{M}_{0}^{j+1}$.

Proof. Suppose $z \in \mathcal{M}_{j}^{s}, s \geq 1$. Then $z$ may be written as a homogeneous polynomial of degree $s$ in the given basis elements for $\mathcal{M}_{j}$, coefficients in $S_{j}$. By grouping the monomials in this expression accordingly, we can write $z=a+b+c$, where $a$ is a homogeneous polynomial of degree $s$ in $x_{i 1}, \ldots, x_{i d}$ whose nonzero coefficients are units in $S_{j} ; b$ is a homogeneous polynomial of degree $s$, each of whose monomials contains at least one $y_{j l}$, and whose nonzero coefficients are units in $S_{j}$; and $c$ is a homogeneous polynomial of degree $s$ whose coefficients are in $\mathcal{M}_{j}$. Thus $c \in \mathcal{M}_{j}^{s+1}$.

Concerning this expression for $z$ we observe, first, that $b \in \mathcal{M}_{0}^{j+1}$-which is clear, since each of its terms contains an element, $y_{j l}, 1 \leq l \leq n-d$, which is in $\mathcal{M}_{0}^{j+1}$. Secondly, $a=0$ if $s<j$. For, noting that units in $S_{j}$ are also units in $S_{0}, a \neq 0$ would yield $a \in \mathcal{M}_{0}^{s} \backslash \mathcal{M}_{0}^{s+1}$; on the other hand $b \in \mathcal{M}_{0}^{j+1} \subset \mathcal{M}_{0}^{s+1}$, and $c \in \mathcal{M}_{j}^{s+1} \subseteq \mathcal{M}_{0}^{s+1}$, so we would get $z=a+b+c \in \mathcal{M}_{0}^{s} \backslash \mathcal{M}_{0}^{s+1}$, a contradiction to 
$z \in \mathcal{M}_{0}^{j} \subseteq \mathcal{M}_{0}^{s+1}$. Finally we see, if $a=0$, that $z \in \mathcal{M}_{0}^{j}$ together with $b \in \mathcal{M}_{0}^{j+1}$ imply $c(=z-b) \in \mathcal{M}_{0}^{j}$; and that, if $a \neq 0$, then $s=j$, so $c \in \mathcal{M}_{j}^{s+1} \subseteq \mathcal{M}_{0}^{s+1}=\mathcal{M}_{0}^{j+1}$.

Now if $s=j$ we set $f:=a$ and $g:=b+c$ to obtain the desired result. In the contrary case we use the observations above, that $c \in \mathcal{M}_{0}^{j} \cap \mathcal{M}_{j}^{s+1}$, to repeat the above procedure, with $c$ in place of $z$, so that we have $c=a_{1}+b_{1}+c_{1}$, where $a_{1}$ is a homogeneous polynomial of degree $s+1$ in the $x_{i m}$ 's, $1 \leq m \leq d$, with unit coefficients; $b_{1}$ is a homogeneous polynomial of degree $s+1$, with unit coefficients in the regular parameters for $\mathcal{M}_{j}$, each term of which includes at least one $y_{j l}$; and $c_{1}$ has coefficients in $\mathcal{M}_{j}$. Now we have $b_{1} \in \mathcal{M}_{0}^{j+1}, c_{1} \in \mathcal{M}_{j}^{s+2}, z=b+a_{1}+b_{1}+c_{1}$, and, again, if $s+1<j$ then $a_{1}=0$.

Upon the $(j-s+1)$ st iteration of this expansion we reach

$$
z=a_{j-s}+\left(b+b_{1}+b_{2}+\cdots+b_{j-s}\right)+c_{j-s} .
$$

Then setting $f:=a_{j-s}$ and $g:=\left(b+b_{1}+\cdots+b_{j-s}\right)+c_{j-s}$ we are done, as $a_{j-s}$ is a homogeneous polynomial of the prescribed sort, as $b_{l} \in \mathcal{M}_{0}^{j+1}$ for all $t$, and as $c_{j-s} \in \mathcal{M}_{j}^{s(j-s+1)}=\mathcal{M}_{j}^{j+1} \subseteq \mathcal{M}_{0}^{j+1}$.

We are finally prepared for the

ProOF OF THEOREM 3.2. For each $i \geq 1$, let $\left\{x_{i 1}, \ldots, x_{i d}, y_{i 1}, \ldots, y_{i, n-d}\right\}$ be as in Proposition 3.7. As Lemma 3.6 shows that the images of $x_{i 1}, \ldots, x_{i d}$ form a basis of $V_{0,1}$, and as $d<n$, we have $V_{0,1} \subset V_{0,0}$, so we can find a $y \in \mathcal{M}_{0}$ such that, for all $i,\left\{x_{i 1}, \ldots, x_{i d}, y\right\}$ is part of a regular system of parameters for $\mathcal{M}_{0}$. We will show that $y \notin$ q.f. $\left(S_{\infty}\right)$ by showing that we cannot write $y=z_{1} / z_{2}$ with $z_{1}, z_{2} \in \mathcal{M}_{\infty}$.

Suppose, by way of contradiction, that $y=z_{1} / z_{2}, z_{1}, z_{2} \in \mathcal{M}_{\infty}$. Since $y \neq$ $0, z_{1} \neq 0$, so there exists a positive integer $r$ such that $z_{1} \in \mathcal{M}_{0}^{r} \backslash \mathcal{M}_{0}^{r+1}$. Since $z_{1} \in \mathcal{M}_{\infty} \subseteq \mathcal{M}_{r}$, we may apply Lemma 3.8, with $i:=r$, to write $z_{1}=f_{1}+g_{1}$ with $g_{1} \in \mathcal{M}_{0}^{r+1}$, and with $f_{1}$ a nonzero homogeneous polynomial of degree $r$ in $x_{r 1}, \ldots, x_{r d}$ whose nonzero coefficients are units in $S_{0}$. On the other hand we have $z_{1}=y z_{2}$, so, since the $\operatorname{ord}_{\mathcal{M}_{0}}$ function is a valuation, with $\operatorname{ord}_{\mathcal{M}_{0}} z_{1}=r$ and $\operatorname{ord}_{\mathcal{M}_{0}} y=1$, we must have ord $\operatorname{M}_{0} z_{2}=r-1$, i.e., $z_{2} \in \mathcal{M}_{0}^{r-1} \backslash \mathcal{M}_{0}^{r}$.

Now let $y_{r-1,1}, \ldots, y_{r-1, n-d} \in \mathcal{M}_{r-1} \cap \mathcal{M}_{0}^{r}$ such that $\left\{x_{r 1}, \ldots, x_{r d}, y_{r-1,1}, \ldots\right.$, $\left.y_{r-1, n-d}\right\}$ is a regular system of parameters for $\mathcal{M}_{r-1}$, as in Proposition 3.7 (here, $j:=r-1$ ). We apply Lemma 3.8 to $z_{2}$, since $z_{2} \in \mathcal{M}_{0}^{r-1} \cap \mathcal{M}_{r-1}$, to write $z_{2}=f_{2}+g_{2}$, where $f_{2}$ is a nonzero homogeneous polynomial of degree $r-1$ in $x_{i 1}, \ldots, x_{i d}$ whose nonzero coefficients are units in $S_{0}$, and where $g_{2} \in \mathcal{M}_{0}^{r}$. Thus $z_{1}=y z_{2}$ yields

$$
f_{1}+g_{1}=y f_{2}+y g_{2}
$$

Consider for a moment the function $\varphi: S_{0} \backslash\{0\} \rightarrow \operatorname{gr}^{\mathcal{M}_{0}}\left(S_{0}\right)$ (the associated graded ring of $S_{0}$ with respect to $\left.\mathcal{M}_{0}\right)$ given by $\varphi(h)=\bar{h} \in \mathcal{M}_{0}^{t} / \mathcal{M}_{0}^{t+1}$, where $h \in \mathcal{M}_{0}^{t} \backslash \mathcal{M}_{0}^{t+1}$. Then $\varphi\left(h_{1} h_{2}\right)=\varphi\left(h_{1}\right) \varphi\left(h_{2}\right)$ (where the multiplication on the right-hand side is that in gr $\left.^{\mathcal{M}_{0}}\left(S_{0}\right)\right)$ because ord $\mathcal{M}_{0}$ is a valuation, and $\varphi\left(h_{1}+h_{2}\right)=$ $\varphi\left(h_{1}\right)+\varphi\left(h_{2}\right)$ if $\varphi\left(h_{1}\right)+\varphi\left(h_{2}\right) \neq 0$ and $h_{1}, h_{2} \in \mathcal{M}_{0}^{t} \backslash \mathcal{M}_{0}^{t+1}$. Applying $\varphi$ to $(*)$ gives us

$$
\varphi\left(f_{1}\right)=\varphi\left(f_{1}+g_{1}\right)=\varphi\left(y f_{2}+y g_{2}\right)=\varphi\left(y f_{2}\right)=\varphi(y) \varphi\left(f_{2}\right)
$$


But, letting

$$
\sum_{u=1}^{e_{1}} m_{u}:=f_{1}, \quad \sum_{v=1}^{e_{2}} m_{v}:=f_{2},
$$

be the expressions for $f_{1}$ and $f_{2}$ as sums of monomials in $x_{i 1}, \ldots, x_{i d}$ whose nonzero coefficients are units in $S_{0}$, we get

$$
\sum_{u=1}^{e} \varphi\left(m_{u}\right)=\sum_{v=1}^{e_{2}} \varphi(y) \varphi\left(m_{v}\right),
$$

a contradiction, since $\varphi\left(x_{i 1}\right), \ldots, \varphi\left(x_{i d}\right), \varphi(y)$ are algebraically independent over $k$ in the polynomial ring $\operatorname{gr}^{\left(\mathcal{M}_{0}\right)}\left(S_{0}\right)$.

The author is grateful to the referee for providing greatly improved versions of the original proofs in this section.

4. Finite chain conditions. We begin by recalling a few results from valuation theory (cf. [12, Appendix 2]).

DEFINITION 4.1. Given a quasilocal domain $(R, \mathcal{M})$, a prime divisor of $R$ is a DVR, $\left(V, \mathcal{M}_{v}\right)$, such that $V>R$ and $\operatorname{restrdeg}_{R} V=\operatorname{dim} R-1$.

PROPOSITION 4.2. Any positive-dimensional local domain $(R, \mathcal{M})$ has a prime divisor.

Proof. [7, Proposition 1].

PROPOSITION 4.3. If $\left(V, \mathcal{M}_{v}\right)$ is any valuation ring birationally dominating the local domain $(R, \mathcal{M})$ then $\operatorname{restrdeg}_{R} V \leq \operatorname{dim} R-\operatorname{rank} V$.

ProOf. [12, Proposition 1, p. 330].

PROpOSITION 4.4. If $\left(V, \mathcal{M}_{v}\right)$ is a prime divisor of $(R, \mathcal{M})$ then $V / \mathcal{M}_{v}$ is a finitely generated field extension of $R / \mathcal{M}$.

Proof. [12, Corollary 2, p. 339].

LEMMA 4.5. If $(S, \mathcal{N})>(R, \mathcal{M})$ are two $n$-dimensional local domains $(n \leq 1)$ then $S / \mathcal{N}$ is a finite algebraic field extension of $R / \mathcal{M}$.

Proof. Using Proposition 4.2, choose a prime divisor $\left(V, \mathcal{M}_{v}\right)$ for $(S, \mathcal{N})$. Then $V>R$ as well, and we have, using Proposition 4.3, that

$$
\begin{aligned}
n-1 & =\operatorname{dim} R-\operatorname{rank} V \geq \operatorname{restrdeg}_{R} V \\
& =\operatorname{restrdeg}_{S} V+\operatorname{restrdeg}_{R} S=n-1+\operatorname{restrdeg}_{R} S .
\end{aligned}
$$

This implies, first, that $\operatorname{restrdeg}_{R} S=0$, i.e., $S / \mathcal{N}$ is algebraic over $R / \mathcal{M}$, and second, that $V$ is a prime divisor of $R$. Then, by Proposition $4.4, V / \mathcal{M}_{v}$ is finitely generated over $R / \mathcal{M}$, which implies that $S / \mathcal{N}$ is also finitely generated over $R / \mathcal{M}$, since $V / \mathcal{M}_{v} \supseteq S / \mathcal{N} \supseteq R / \mathcal{M}$.

Now we are ready to prove the main result of this paper. We denote by $R(R)$ the set of RLR's properly containing a local domain $R$, having the same dimension as $R$, and contained in the quotient field of $R$. We order this set by inclusion. 
THEOREM 4.6. For any local domain $(R, \mathcal{M})$ of positive dimension, $R(R)$ satisfies d.c.c.

Proof. Let $\left(S_{0}, \mathcal{M}_{0}\right) \supseteq\left(S_{1}, \mathcal{M}_{1}\right) \supseteq \cdots \supseteq R$ be a descending chain of elements of $R(R)$. By Note 2.1 , we have $S_{i}>R$ for all $i$, so $S_{0} / \mathcal{M}_{0} \supseteq S_{1} / \mathcal{M}_{1} \supseteq \cdots$ is a descending chain of finite algebraic field extensions of $R / \mathcal{M}$ by Lemma 4.5 . Therefore this chain of residue fields must stabilize. If the chain of RLR's fails to stabilize then it has infinitely many different rings, all dominating $R$, and with stabilizing residue field, the situation to which Theorem 3.2 applies. Then Theorem 3.2 tells us that q.f. $(R) \subseteq$ q.f. $\left(S_{\infty}\right) \subset$ q.f. $\left(S_{0}\right)=$ q.f. $(R)$, a contradiction.

DEFINITION 4.7. By a minimal $R L R$ over $R$ we mean a minimal element of $R(R)$.

COROLLARY 4.8. If $S>R$ is a pair of $n$-dimensional $R L R$ 's, then any chain of $R L R$ 's $\left\{S_{\lambda}\right\}$, between $R$ and $S$ must contain a minimal element, $S^{\prime}, S_{\lambda}>S^{\prime}>R$ for all $\lambda$.

PROOF. Otherwise we would be able to find an infinite strictly descending chain in $R(R)$, contrary to Theorem 4.6

This corollary shows that birational RLR's have a nice structure. That the structure is not so nice in more general classes of local rings is shown by the following example.

EXAMPLE 4.9. Let $k$ be any field. Let

$$
\begin{gathered}
R:=k[X, Y]_{(X, Y)}, \quad S:=k[X, Y, Y / X]_{(X, Y / X)}, \\
S_{i}:=k\left[X, Y, Y^{i+1} / X\right]_{\left(X, Y, Y^{i+1} / X\right)}, \quad i \geq 1 .
\end{gathered}
$$

Then $S>S_{1}>S_{2}>\cdots>R$. Since a local domain caught between two local domains of dimension $n$ must also have dimension $n$ (see [9, Lemma 5.3]), $\operatorname{dim} S_{i}=$ 2 for all $i$. Since

$$
S_{i} \cong k[X, Y, Z]_{(X, Y, Z)} /\left(X Z-Y^{i+1}\right) k[X, Y, Z]_{(X, Y, Z)},
$$

it is easily verified that $S_{i}$ satisfies Serre's conditions $S_{2}$ and $R_{1}$, so that $S_{i}$ is normal (cf. [5, Theorem 39]) and hence Cohen-Macaulay, since $\operatorname{dim} S_{i}=2$ [12, Example (3), p. 397].

Moreover, since any 2-dimensional normal local ring birationally dominating a 2-dimensional RLR has a rational singularity [4, Proposition (1.2)], each $S_{i}$ must have a rational singularity.

Again restricting our attention to RLR's, however, and using a related result of Shannon's, we are able to obtain the following corollary. This corollary answers a question raised by Shannon [9, Remark 3.8].

COROLLARY 4.10. If $S>R$ is a pair of n-dimensional RLR's then any chain between them is finite in length.

PROOF. Let $\left\{S_{\lambda}\right\}$ be a chain of RLR's between $R$ and $S$. If $S$ is minimal over $R$, then $S>R$ is the only such chain, and is clearly finite. Otherwise, use Corollary 4.8 to pick $S_{1} \in\left\{S_{\lambda}\right\}$ minimal over $R, S>S_{\lambda}>S_{1}>R$ for all $\lambda$. If $S$ is minimal over $S_{1}$, we are done; otherwise pick $S_{2}$ minimal over $S_{1}, S>S_{\lambda}>S_{2}>S_{1}>R$ for all $\lambda$. Either there eventually exists an $N$ such that $S$ is minimal over $S_{N}$, so that $S>S_{N}>S_{N-1}>\cdots>S_{1}>R$ forms a saturated chain, or else we 
obtain an infinite ascending chain of RLR's between $R$ and $S$. That this latter situation cannot hold was shown by Shannon [9, Proposition 5.1]. Thus the chain must become saturated after finitely many steps.

Note, however, that two saturated chains need not have the same length, as is illustrated in the following 3-dimensional example, due to Shannon [9, Remark 3.8].

EXAMPLE 4.11 . Let $k$ be any field, and set

$$
\begin{aligned}
& R_{0}:=k[X, Y, Z]_{(X, Y, Z)}, \\
& \left.R_{1}:=k[X, Z, Y / X]\right)_{(X, Z, Y / X)}=R_{0}[Y / X]_{\left(\mathcal{M}_{0} R[Y / X], Y / X\right)}, \\
& R_{2}:=k[X, Y / X, Z / X]_{(X, Y / X, Z / X)}=R_{1}[Z / X]_{\left(\mathcal{M}_{1} R_{1}[Z / X], Z / X\right)}, \\
& R_{3}:=k[X, Y / X, Z / Y]_{(X, Y / X, Z / X)}=R_{2}[Z / Y]_{\left(\mathcal{M}_{2} R_{2}[Z / Y], Z / Y\right)} .
\end{aligned}
$$

Then $R_{0}<R_{1}<R_{2}<R_{3}$, each $R_{i}$ is a RLR of dimension 3, and each $R_{i}$ is obtained from $R_{i-1}$ by a simple monoidal transformation, so this chain is saturated (because simple monoidal transforms of $R$ are minimal over $R$-see [8, Theorem 5.1]), and has length 3 . On the other hand, letting

$$
\begin{aligned}
& S_{1}:=k[X, Y, Z / Y]_{(X, Y, Z / Y)}=R_{0}[Z / Y]_{\left(\mathcal{M}_{0} R_{0}[Z / Y], Z / Y\right)}, \\
& S_{2}:=k[X, Y / X, Z / Y]_{(X, Y / X, Z / Y)}=S_{1}[Y / X]_{\left(\mathcal{M}_{S_{1}} S_{1}[Y / X], Y / X\right)}=R_{3}
\end{aligned}
$$

then $R_{0}<S_{1}<S_{2}=R_{3}$ is again a saturated chain of RLR's, each a simple monoidal transform of the previous one. Though these two chains both lie between $R_{0}$ and $R_{3}=S_{2}$, they have different lengths.

QUESTION 4.12. Given two RLR's of dimension $n, S>R$, does there exist a uniform upper bound, $N$, depending only on $S$ and $R$, such that every saturated chain of RLR's between $S$ and $R$ has length $\leq N$ ?

In relation to this question, we should note that Hironaka has apparently shown that a similar question for projective models has an affirmative answer. Namely, if $X$ and $Y$ are two $n$-dimensional, birational normal projective models such that $Y$ dominates $X$, then any birational sequence of normal models between $X$ and $Y$ has finite length, bounded uniformly by a fixed number depending only on $X$ and $Y$ (see [9, Remark 3.8]).

5. Some related questions. In this section we discuss some applications and directions for further research. One immediate question is the following.

QUESTION 5.1. Are RLR's the broadest class of local domains having the property that, given two rings, $S>R$, in this class, any chain of rings in this class between $S$ and $R$ must be finite in length?

Corollary 4.10 says that RLR's have this property in any dimension, while Example 4.9 shows that the class of Cohen-Macaulay local domains, the class of normal local rings, and the class of rational singularities do not have this property, even in dimension 2.

Another generalization of RLR's is the class of normal local rings whose order function is a valuation. The following example of Shannon's [9, Example 3.9] shows that this class also fails to have this property of RLR's.

EXAMPLE 5.2. Let $k$ be a field of characteristic 0 ,

$$
(R, \mathcal{M}):=\left(k[X, Y]_{(X, Y)},(X, Y) R\right)
$$


and for $i \geq 1$,

$$
\begin{aligned}
R_{i} & :=k\left[X, Y, Y^{2} / X, \ldots, Y^{i+1} / X^{i}\right]_{\left(X, Y, Y^{2} / X, \ldots, Y^{i+1} / X^{i}\right)}, \\
S & :=k[X, Y / X]_{(X, Y / X)} .
\end{aligned}
$$

Then we have $R<R_{1}<R_{2}<\cdots<S, R$ and $S$ are RLR's of dimension $2, S$ is a quadratic transform of $R, R_{i}$ has dimension 2 for all $i$ (since $R<R_{i}<S$-see [9, Lemma 5.3]), $R_{i}$ is normal for all $i$ (since $k\left[X, Y, Y^{2} / X, \ldots, Y^{i+1} / X^{i}\right]$ is the integral closure of $k\left[X, Y^{i+1} / X^{i}\right]$ in $k(X, Y)-c f$. [2, Theorem 7]), and this is a strictly ascending chain. Furthermore, ord $\mathcal{M}_{i} \mid R_{i}$ (where $\left.\mathcal{M}_{i}:=\left(X, Y, Y^{2} / X, \ldots, Y^{i+1} / X^{i}\right) R_{i}\right)$ $=\operatorname{ord}_{\mathcal{M} \mid R_{i}}$, since if $\left(V, \mathcal{M}_{v}\right)=$ the valuation ring of the valuation ord $\mathcal{M}_{\mathcal{M}}, \mathcal{M}_{v}^{n} \cap R_{i}=$ $\mathcal{M}_{i}^{n}$ for all $n$, which implies that $\operatorname{ord}_{\mathcal{M}}=\operatorname{ord}_{\mathcal{M}_{i}}$ and so ord $\mathcal{M}_{i}$ is a valuation. This gives infinitely many normal local rings, each of whose ord function is a valuation, between two RLR's, so Corollary 4.10 does not extend to this class of rings.

Corollary 4.8 implies that every $n$-dimensional $\operatorname{RLR},(S, \mathcal{N})$, birationally dominating a given $n$-dimensional $\mathrm{RLR},(R, \mathcal{M})$, must contain a minimal RLR over $R$. It was proven by J. Sally [8, Theorem 5.1] and by D. Shannon [9, Proposition 3.7] that if $n>1$, simple monoidal extensions of $R$ are minimal elements of $R(R)$. If $n>2$, another type of minimal extension was given by J. Sally [8, Theorem 5.1], namely an extension of the form $R[a / b]_{\mathcal{N}}$, where $a, b \in R$ are relatively prime, $a \neq 0 \neq b, a \in \mathcal{M} \backslash \mathcal{M}^{2}, b \in \mathcal{M}^{2},(a, b) R$ is a prime ideal, and $\mathcal{N}$ is any height $n$ maximal ideal of $R[a / b]$. We denote this type of extension as a "Sally" extension. Example 5.4 below, due to $C$. Huneke, shows that simple monoidal extensions and Sally extensions are not the only minimal elements of $R(R)$, if $R$ is an arbitrary RLR. We need a preliminary lemma.

LEMMA 5.3. If $(S, \mathcal{N})$ and $(R, \mathcal{M})$ are $n$-dimensional $R L R ' s, n>2$, and $S$ is either a simple monoidal extension or a Sally extension of $R$, then $\mathcal{M} S$ requires at most $n-1$ generators.

ProOF. Suppose $\mathcal{M}=\left(x_{1}, \ldots, x_{n}\right) R$, where $S=R\left[x_{2} / x_{1}\right]_{Q}$ (if $S$ is a simple monoidal extension) or $S=R\left[x_{1} / b\right]_{Q}$ (if $S$ is a Sally extension). Clearly, in the first case, $\mathcal{M} S=\left(x_{1}, x_{3}, x_{4}, \ldots, x_{n}\right) S$ is $(n-1)$-generated. In the second case, $\mathcal{M} S=\left(x_{2}, x_{3}, \ldots, x_{n}, b\right) S$, but since $b \in \mathcal{M}^{2} \subseteq \mathcal{N}^{2}$, we have $\mathcal{M} S=\left(x_{2}, x_{3}, \ldots, x_{n}\right) S$ by Nakayama's lemma.

EXAMPLE 5.4. Let $S:=k[X, Y, Z]_{(X, Y, Z)}$, where $k$ is any field,

$$
R:=k\left[X Y Z, Y^{2} Z, X^{3} Z\right]_{\left(X Y Z, Y^{2} Z, X^{3} Z\right)} \text {. }
$$

Then $R$ and $S$ are 3-dimensional RLR's and since

$$
\operatorname{det}\left(\begin{array}{lll}
1 & 1 & 1 \\
0 & 2 & 1 \\
3 & 0 & 1
\end{array}\right)=-1
$$

we have $S>R$. If there existed a simple monoidal or Sally extension, $T$, of $R, S>$ $T>R$, then by Lemma $5.3, \mathcal{M} T$ would be at most 2 -generated, so $\mathcal{M} S=(\mathcal{M} T) S$ would be at most 2-generated. But $\mathcal{M} S=\left(X Y Z, Y^{2} Z, X^{3} Z\right) k[X, Y, Z]_{(X, Y, Z)}$ is 3 -generated for, if it were generated by less than three elements, then, since we are in a local ring, one such smaller generating set would have to be a subset of $\left\{X Y Z, Y^{2} Z, X^{3} Z\right\}$, but none of these three elements lies in the ideal generated 
by the other two in $S$. On the other hand, Corollary 4.8 above states that there must exist some minimal element of $R(R)$ between $S$ and $R$ (it might be $S$ ). Thus there must exist at least one new type of minimal regular local overring. Specific calculations show that in fact $S$ is minimal over $R$.

\section{REFERENCES}

1. S. Abhyankar, On the valuations centered in a local domain, Amer. J. Math. 78 (1956), 321-348.

2. _ Uniformization of Jungian local domains, Math. Ann. 159 (1965), 1-43.

3. W. Krull, Idealtheorie, Springer-Verlag, Berlin and New York, 1968.

4. J. Lipman, Rational singularities, with applications to algebraic surfaces and unique factorization, Inst. Hautes Etudes Sci. Publ. Math. 36 (1969), 195-279.

5. H. Matsumura, Commutative algebra, (2nd ed.), Benjamin/Cummings, Reading, Mass., 1980.

6. M. Nagata, Local rings, Interscience, New York, 1962.

7. M. Sakuma, Existence theorems of valuations centered in a local domain with preassigned dimension and rank, J. Sci. Hiroshima Univ. 21 (1957), 61-67.

8. J. Sally, Regular overrings of regular local rings, Trans. Amer. Math. Soc. 171 (1972), 291-300.

9. D. Shannon, Monoidal transforms of regular local rings, Amer. J. Math. 95 (1973), 294320.

10. O. Zariski, Reductions of the singularities of algebraic three dimensional varieties, Ann. of Math. (2) 45 (1944), 472-542.

11. O. Zariski and P. Samuel, Commutative algebra, vol. I, Van Nostrand, Princeton, N. J., 1958.

12. _ Commutative algebra, vol. II, Van Nostrand, Princeton, N. J., 1960.

Department of Mathematics, Purdue University, West lafayette, Indiana 47907

Current address: Department of Mathematics, University of Utah, Salt Lake City, Utah 84112 